\section{e-Journal of Educational \\ Research, Assessment and Evaluation} \section{REIIEVE}

Revista ELectrónica de Investigación y EValuación Educativa

\title{
ELABORACIÓN UN INSTRUMENTO PARA CONOCER LAS CARACTERÍSTICAS DE LA BANDA SONORA DE LA PROGRAMACIÓN INFANTIL DE TELEVISIÓN
}

\section{[Development of an instrument for analyzing the characteristics of the soundtracks of children's television programmes]}

\author{
by / por \\ $\underline{\text { Article record }}$ \\ $\underline{\text { About authors }}$ \\ HTML format \\ Amparo Porta (porta@edu.uji.es) \\ Reina Ferrández (ferrande@edu.uji.es) \\ Ficha del artículo \\ $\underline{\text { Sobre los autores }}$ \\ Formato HTML
}

\begin{abstract}
Television is one of the most important elements in the construction of the musical and audible reality of children and teenagers. It offers a selected and edited version of a musical environment, with an indisputable level of communicative and patrimonial influence. This paper is part of a research project that sets out to know what children are listening to in children's television programming, in a sample of Latin American television coverage. The general objective has been to establish the parameters of everyday listening experiences and to create an instrument which is able to determine the objective elements of listening environments through their most notable medium: the soundtrack of children's television programmes. Here we present the creation and validation process of such an instrument. The more important elements are the consensus process, since we attempt to validate the instrument within the Latin-American context and decision-making because of the inherent characteristics of the music as variable analysed, since sound develops over time, and this makes an accurate classification of it very difficult.
\end{abstract}

\section{Keywords}

Soundtrack, quantitative analysis, validation, observation, classification, television, listening.

\section{La banda sonora}

Los cuatro componentes sónicos, música, sonido, silencio y escucha, han sido explica-

\section{Resumen}

La televisión es un elemento destacado en la construcción de la realidad musical y sonora del niño y del adolescente que ofrece, como totalidad, una versión seleccionada y editada del entorno musical. El artículo que presentamos forma parte de un proyecto de investigación que trata de conocer qué escuchan los niños en la programación infantil de televisión en una muestra latinoamericana. El objetivo general ha sido establecer los parámetros de la escucha televisiva y determinar sus elementos objetivos. Lo que presentamos en este artículo es el proceso de elaboración y validación de dicho instrumento. El proceso ha tenido como elementos generadores el consenso por el ámbito de aplicación con validez latinoamericana y la toma de decisiones, por la naturaleza de la música como variable de análisis y las características del sonido, cuyo desarrollo en el tiempo requiere formas específicas de clasificación.

\section{Descriptores}

Banda sonora, análisis cuantitativo, validación, observación, clasificación, televisión, escucha. 
Reyes, 2006). Mucho menos lo han sido desde la Comunicación y prácticamente nada desde la educación general y musical. Sin embargo, podemos decir que conceptos tan familiares cuando hablamos del sonido en términos de los Mass Media como: sonido grabado, bucles, simultaneidad y manipulación sonora, edición, cambios de velocidad, lenguaje fonético expresado por objetos; y otros, como estirar, comprimir, voltear, manipular, han formado parte del arte de las vanguardias del S. XX.

Diferentes trabajos sobre la influencia de la televisión como los de Silverstone, 1996; Morley, 1996; García Muñoz, 1997, Vargas y Barrena, 2003; han sido realizados en contextos variados, y de manera más específica los estudios de la televisión infantil en España han sido realizados por Vallejo-Nágera, 1987; Ferrés, 1994; Orozco, 1996; Aguaded, 2005; De Moragas, 1991; Reig, 2005. Estos autores han estudiado los hábitos televisivos de jóvenes y adolescentes insistiendo en que en muchas ocasiones pueden ser preocupantes. De igual modo Pintado considera que la televisión, desde su integración en el mercado, más que fabricar contenidos lo que principalmente fabrica son audiencias (Pintado, 2006). De todos los estudios consultados, el Informe Pigmalión sobre el impacto de la televisión en la infancia realizado (Del Río, Álvarez, y Del Río, 2004), aporta gran información sobre España y permite articular una visión global con el fin de integrar el problema de la escucha televisiva en una perspectiva cultural del desarrollo infantil. Estos autores defienden, entre otras muchas cuestiones, crear a largo plazo una programación televisiva infantil que tenga como referentes las propuestas culturales.

Nuestro tema de interés es la música desde el lado de la escucha, cuya presencia televisiva se produce mediante la banda sonora de las programaciones infantiles de televisión. Son escasos los trabajos encontrados en torno al estudio cognitivo, social y comunicativo de la música en la televisión, pese a su influencia como modelador y constructor de la realidad del niño y el adolescente. El sonido es parte del sistema auditivo y uno de los primeros sentidos en habilitarse en los humanos. La escucha de la banda sonora de la televisión en España tiene unas repercusiones indiscutibles, especialmente si consideramos que nuestro país es el segundo europeo en el ranking del consumidor televisivo, con una media de 218 minutos de exposición televisiva al día, tal como indica el Libro Blanco: La educación en el entorno audiovisual, 2003 elaborado por el Consejo audiovisual de Cataluña (CAC). Desde la vertiente cognitiva, el informe Pigmalión (Del Río, Álvarez, y Del Río, 2004) sin hacerlo de forma explícita, involucra a la música cuando habla de conceptos como atención, ritmo, densidad sonora, necesidad de estilos más lentos y equilibrados, reflexividad, alfabetización audiovisual. El informe realiza tres recomendaciones: 1) Investigación y búsqueda de alternativas, 2) Inclusión de contenidos sobre alfabetización mediática y 3) Reorganización del currículum educativo (Del Río; Alvarez, y Del Río, 2004) hablan de la dieta televisiva como acumulación de contenidos que producen efectos buscados o primarios, como los de la publicidad, el entretenimiento o la información; y efectos secundarios, provocados intencionalmente, como los educativos o infantiles diseñados. Sus consecuencias, según el informe, son: efectos sobre la atención, la escolaridad, la lectoescritura y la narratividad, entre otros.

\section{La televisión como construcción de la realidad musical y sonora}

La televisión se ha convertido para el niño actual, junto a su experiencia directa, en un importante medio de construcción de la realidad, en el que la creciente mezcla entre los géneros de realidad y ficción le plantea problemas nuevos (Del Río, Álvarez y Del Río, 2004). La música forma parte de la construcción de ese mundo interior y la hibridación se produce por medios sonoros de manera 
efectiva, porque el mundo, en muchas ocasiones, es sustituido por la música y las cosas por las cualidades del sonido (Porta, 2007).

El informe Pigmalión (Del Río, Álvarez, y Del Río, 2004) establece cómo la adicción a la televisión no genera por sí sola competencias audiovisuales salvo de bajo nivel. Las competencias alfabéticas de alto nivel se benefician de una enseñanza articulada entre la alfabetización verbal, audiovisual, y otras alfabetizaciones. Por ello se hace necesaria una investigación que sitúe de forma objetiva los elementos de la escucha presentes en la dieta televisiva como uno de los componentes de sentido, y todo ello tiene una asignatura pendiente en los diseños curriculares. Sólo una alfabetización que contemple los diferentes lenguajes y formas de expresión, entre los que se encuentra la música, podrá abordar los cambios de las formas de representación surgidas de los lenguajes contemporáneos.

Nuestro tema se interesa por el significado. Por ello nos preguntamos si la banda sonora de la televisión infantil se deja reducir a modelos y sistemas semióticos, o quedan siempre resquicios, intersticios y fisuras por donde el sentido -como signo que indica un referente- se filtra o se acumula y se estanca. Juan Reyes llama a estas fisuras Islas de Entropía (Reyes, 2006). Desde un terreno próximo, el de la educación, Vigostky habla de la construcción social de la conciencia (Vygotsky, 1981:162). La respuesta, que rebasa con mucho el objetivo de este artículo, es de orden semiótico porque nos interesa el significado y el sentido de la música que escuchan los niños del entorno más cotidiano de la cultura hispánica: la televisión. Todo ello requiere de un proceso investigador que comienza por la creación de una plantilla de la escucha, hasta ahora inexistente, para después dar un paso adelante, seleccionar las muestras para su aplicación, interpretarla y así proporcionar las alternativas educativas de un entorno sonoro, ya para siempre cambiante.
En este apartado estamos revisando la bibliografía que gira en torno al fenómeno poliédrico de la escucha del entorno cotidiano. Este hábitat sonoro está inscrito en la música popular contemporánea y sus límites se perciben difusos e imbricados en diferentes espacios comunicativos. Sin embargo, desde un nivel descriptivo, podemos decir que actualmente esta música de la cotidianidad es transmitida de forma oral no escrita, utiliza muchas veces una lengua extranjera y su difusión es masiva a culturas heterogéneas. Tiñe todo el contorno comunicativo del cambio del milenio e incorpora los elementos de la ingeniería sonora y de la comunicación. Su importancia se basa en la selección y ordenación de los elementos del lenguaje musical, o dicho de otro modo, en la combinación entre lo que dice, lo que no dice, cómo, cuándo y a quien lo dice (Porta, 2004b).

La complejidad del entorno y su perfil altamente multidisciplinar nos obliga, como primera tarea, objeto de este texto, a la selección de nuestro campo de estudio, y de nuevo vuelve a nosotros la pregunta de investigación ¿Cómo saber lo que escuchan los niños en la televisión.

\section{Necesidad de plantillas de análisis}

\section{Instrumentos para la escucha del entorno sonoro}

Los diferentes estudios sobre la televisión infantil revelan contenidos televisivos con un alto nivel de exposición que utilizan estrategias publicitarias de carácter seductor y poseen un poder de influencia comunicativa indiscutible a través de la suma de sus lenguajes. El informe Pigmalión (Del Río; Álvarez y Del Río, 2004) indica en sus conclusiones una masa crítica inexistente en España pero con potencial en la industria, profesionales audiovisuales e investigadores, y todo ello se incrementa cuando hablamos de banda sonora. La sistematización necesaria para fundamentar nuestro trabajo muestra un gran vacío cuando hablamos del contexto sonoro del niño. Los estudios encontrados, creados 
en su mayoría como experiencias de laboratorio, acotan realidades artificiales para grupos determinados por rasgos de aprendizaje o población escolar de riesgo (Burnard, 2008; Ward-Steinman, 2006); hábitos alimentarios y de salud, vinculando música, publicidad y consumo (Ostbyeit, 1993; Koivusilta, 1999); relación de la música con habilidades de lectura (Register, 2004), relación con la violencia, (Peterson y Newman, 2000) y otros. La necesidad de instrumentos objetivos pasa por la creación de escalas de medida para un nuevo entorno sonoro. Sin embargo, el problema de los instrumentos de medida en música no es nuevo, y este interrogante se agudiza cuando ampliamos el concepto al entorno sonoro cotidiano.

Algunos arquitectos se han manifestado sobre la peculiaridad y necesidad de atención al entorno sonoro, y dicen que cada fragmento del tejido urbano posee unos rasgos sonoros característicos que nos hablan de sus cualidades espaciales, de las temporalidades y de los usos que lo habitan. Por ello dicen que estos rasgos constituyen su identidad ordinaria, cotidiana. Así, pues, necesitamos recurrir a una nueva escucha musical del entorno cotidiano y para ello hemos de apoyarnos en el lenguaje y en los instrumentos de análisis que la propia disciplina musical ha desarrollado (Atienza, 2004).

En los últimos 40 años, diferentes esfuerzos interdisciplinares han trabajado en esta travesía de los saberes y disciplinas. Durante las décadas de 1960 y 1970, dos acercamientos diferentes al análisis sonoro verán la luz. Por una parte, el objeto sonoro de Pierre Schaeffer parte de un análisis fenomenológico del universo audible centrado sobre los modos de percepción auditiva (Schaeffer, 1966). Por otra, la noción de paisaje sonoro de Robert Murray Schafer habla de la representación del entorno sonoro, que podríamos calificar de «compositiva» y se pregunta por nuevas soluciones para nuevas necesidades, y dice que actualmente se está necesitando una revolución equivalente entre los variados campos de los estudios sonoros. La revolución consistirá en la unificación de aquellas disciplinas que se ocupan de la ciencia del sonido y aquellas que se ocupan del arte del sonido. El resultado será el desarrollo de las interdisciplinas ecología acústica y diseño acústico (Schafer, 1977). Sin embargo, Atienza se muestra crítico e indica que el objeto sonoro, destinado a constituir la unidad elemental de un «solfeo sonoro», carece de la escala adecuada para el análisis de configuraciones tan complejas como las urbanas (Atienza, 2004). El concepto de paisaje sonoro presenta igualmente ciertas limitaciones para el análisis del contexto urbano, que encuentran esta vez su origen en una escala de trabajo excesivamente extensa. (Augoyard y Torgue, 1995).

Desde nuestra posición educativa, de acuerdo con Atienza, queremos destacar que el efecto sonoro no es un objeto en sí, ni se ocupa de la materia sonora en cuanto objeto, sino en cuanto medio. Persiste pues la interrogación acerca del propio sonido, abordada por Pierre Schaeffer y Robert Murray Schafer. No se trata aquí de estudiar el sonido como finalidad en sí mismo, desvinculado de todo contexto, al contrario, el estudio se realiza en función de un tiempo y espacio precisos. Es, precisamente, Jean-François Augoyard y el laboratorio Cresson (Augoyard y Leroux, 1989) quienes se presentan como herederos y complementarios de las dos anteriores. Esta línea investigadora surge a partir de la necesidad de encontrar una herramienta interdisciplinar adecuada a la escala de una configuración urbana que permita integrar otras dimensiones que la puramente estética (Atienza, 2004)). Este concepto se presenta como medio de descripción cualitativa de la experiencia sonora cotidiana. El efecto describe los vínculos existentes entre las dimensiones física y humana del entorno, entre el espacio sonoro, nuestra percepción y el modo en que lo representamos. El laboratorio Cresson de Grenoble establece un protocolo de encuesta en el que indica un cambio de paradigma que reposa sobre tres tipos 
de consignas básicas: consignas relativas a la descripción, consignas relativas al recorrido y consignas relativas a la experiencia. Los trabajos de observación de este laboratorio han utilizado unos parámetros que han sido ampliados e incluyen los datos contextuales y también más privados, los experienciales, de lo sonoro. Así, pues, no hablamos sólo de música o arte sonoro, añadimos además "situado”. Pero, para comprender de qué modo puede ser percibida esta materia sonora situada, necesitamos entender primero cuáles son las cualidades sonoras que dichas configuraciones poseen. La escucha de la televisión es, por tiempo de exposición, la mayor del hábitat sonoro contemporáneo. Las músicas televisivas están construidas con una organización musical adaptada al medio y una selección estilística basada en estrategias publicitarias e importaciones musicales de las culturas industriales dominantes norteamericanas o altamente competitivas de los manga japoneses. $\mathrm{Y}$, en este contexto, los contenidos de la proximidad (De Moragas, 1991) y la escucha como valor educativo comunicativo, estético y patrimonial, tal y como proclama la normativa española en sus diferentes leyes (BOE, 2003, 2006a y 2006b) parecen quedar en entredicho.

Este es el marco general de nuestro objeto de estudio, pero la investigación empírica requiere el uso de instrumentos. Su contenido destacado es la escucha del hábitat sonoro contemporáneo de procedencia industrial, alto impacto y refuerzo, así como utilización de fórmulas musicales, sonoras y comunicativas adaptadas al medio televisivo. De esta forma justificamos desde la educación musical la necesidad de creación de un instrumento de medida de la escucha, todavía inexistente, que permita objetivar la búsqueda de alternativas educativas. Para ello es necesario realizar un diagnóstico de los contenidos de mayor refuerzo, impacto y continuidad de la banda sonora televisiva y sus ausencias, tan determinantes como las presencias.
Los resultados de esta investigación pueden servir de orientación a las audiencias, productoras, cadenas, y educadores. De otro lado, la necesidad de políticas para la preservación, generación y mantenimiento de macro y micro-contextos es necesaria para adaptar continuamente el entorno diario infantil a sus mejores potenciales, que corresponden a los contenidos de la proximidad. (De Moragas, 1991; Porta, 2004b). Finalmente consideramos que el estudio será de utilidad para futuras reconstrucciones de los diseños curriculares.

\section{Recorridos, secuencias y líneas de tiempo}

La música se produce en el tiempo, por ello nuestro instrumento ha de tener un anclaje temporal doble: qué se escucha y dónde está situado aquello que se escucha. La primera cuestión a abordar es la acotación de la secuencia para ser observada y analizada de forma objetiva, sin ambigüedades. El protocolo de encuesta del laboratorio Cresson, desde la arquitectura, nos orienta por medio de la definición de tres ámbitos de la observación de la escucha: descripción, recorrido y experiencia. En nuestro caso vamos a delimitar los dos primeros para así poder acceder a algunas de las características que componen el complejo entramado cognitivo, social, cultural y afectivo de la experiencia auditiva.

DESCRIBIR: Qué se escucha

EL RECORRIDO: En qué momento se escucha

EXPERIENCIA: Desde dónde se escucha (Porta, 2007)

\section{El método y codificación}

Este trabajo es parte de una investigación más amplia que tiene como objetivo conocer qué escuchan los niños en una muestra televisiva de la cultura hispánica. El marco teórico de referencia para su interpretación, de carácter semiótico, utiliza el modelo de Eco y Cantarell, (1978). Umberto Eco, en su introducción a La Estructura Ausente dice que 
una investigación semiótica solamente tiene sentido si la estructura del campo es asumida como una entidad imprecisa que el método se propone aclarar (provocando continuamente sus contradicciones). No tiene sentido si la estructura establecida por deducción se considera "verdadera”, “objetiva” y "definitiva”. Por ello propone como metodología:

\section{Cotejar definiciones}

2. Establecer el campo semiótico en términos de vastedad y desorden

3. Crear un modelo de investigación sometido a contradicción

De esta forma -dice- si la operación tuviera éxito se lograría algo que casi suena utópico: "mantener la complejidad del campo confiriéndole una estructura, y por lo tanto, transformar el campo en un sistema.

Así, pues, siguiendo a Eco, nuestra hipótesis operativa es: transformar el campo, la banda sonora de la infantil, en un sistema de plantilla de la escucha.

En La Estructura ausente (Eco y Cantarell, 1978), Umberto Eco dice que el estudio de la comunicación de masas se propone un objeto unitario en la medida en que postula que la industrialización de la comunicación ha de cambiar, no solamente las condiciones de recepción y emisión del mensaje, sino también el propio sentido del mensaje (es decir aquella masa de significados que se creía que era una parte inalterable del mensaje, tal como lo había pensado el autor, independientemente de sus modos de difusión).

Así, pues, tal como hemos justificado, el marco teórico general de la investigacion, de carácter semiótico, es el propuesto por Umberto Eco (Eco y Cantarell, 1978), y como específico para la construcción de la plantilla de la escucha, utilizaremos como modelo el propuesto por Ariza (Gómez-Ariza, 2000).

\section{El modelo}

Los modelos que intentan explicar la manera en que la música está representada men- talmente, varían en el rango de fenómenos que explican y en las unidades representación que postulan (Gómez Ariza, Bajo, Puerta-Melguizo y Mazizo, 2000). Uno de los primeros problemas para juzgar estas teorías o proponer otras nuevas, está en delimitar cuáles son las características de la música que determinan la manera en la que percibimos y, posteriormente, la recordamos. Sin embargo, hay pocos trabajos que, de forma sistemática, revisen cada uno de de los factores que componen la Música y la manera en que influyen en la percepción y el recuerdo de la información musical. Una de las dificultades para realizar este tipo de revisión, según Gómez-Ariza, es que no existe un marco teórico unificador que permita organizar los datos existentes. En nuestro caso, para conocer lo que escuchan los niños en la televisión, hemos partido del modelo elaborado por estos autores. En su revisión de los datos empíricos y las explicaciones teóricas delimitan la importancia de distintas características musicales en la manera en que percibimos y recordamos piezas musicales. Consideran que los estímulos musicales están compuestos de un gran número de dimensiones que se combinan para crear el patrón auditivo complejo que forma la música. Parten del supuesto de que la música y el lenguaje ofrecen similitudes en su estructura y que, por lo tanto, se pueden agrupar las distintas variables musicales en aquellas que hacen referencia a la combinación de sonidos (fonología), a la segmentación y las reglas organizativas (sintaxis) y al significado de la música (semántica). En este planteamiento, el contorno, los intervalos y la tonalidad son variables que determinan la combinación de los sonidos que componen la música (fonología musical), el patrón rítmico, los acentos y las reglas de formación son esenciales para realizar procesos de segmentación de una pieza musical (sintaxis musical). Finalmente, la existencia de jerarquías tonales estructuran, organizan la música y generan expectativas musicales. Estas últimas se consideran fundamentales en la escucha e interpretación de la música y, junto a otros factores expre- 
sivos como el tempo o el timbre, parecen generar respuestas afectivas a la música (semántica de la música).

La estructura propuesta por el modelo nos permite la interpretación de la escucha de la televisión en los niños. Desde este marco hemos confeccionado una tabla basada en las categorías de Gómez-Erquiza para el estudio de la escucha de la televisión infantil en una muestra latinoamericana.

\section{Procedimiento}

El proceso de validación del instrumento que presentamos se ha realizado en 4 fases:

a. la construcción de la plantilla piloto

b. validación mediante el juicio de expertos
c. segunda plantilla
d. validación interjueces

\section{Construcción de la Plantilla piloto}

Para la elaboración de la plantilla de observación piloto sobre las cualidades sonoras se consideraron sus características más representativas susceptibles de medición. De esta forma, se establecieron las siguientes variables de análisis:

1. Sin música

2. Con música

2.a. Tipo de sonido

2.b. Voz

2.c. Ritmo

2.d. Tipo de comienzo

2.e. Intensidad

2.f. Agógica

2.g. Género y estilo

2.h. Tonalidad

2.i. Modo

2.j. Estructura musical

2.k. Simultaneidad sonora

Cada una de estas variables contenía diferentes subdivisiones, hasta un total de 67 características diferentes. Esta plantilla de observación fue modificada por medio de un juicio de expertos. Las revisiones a la planti- lla inicial propuesta fueron realizadas en el II Encuentro investigador sobre la banda sonora de la televisión infantil y juvenil en el ámbito latinoamericano. Variables, impacto $e$ influencia en el patrimonio sonoro", celebrado en la Universidad de LANUS, Buenos Aires (Argentina) entre el 14 y 18 de Mayo de 2008. En diferentes sesiones de trabajo se realizó un ajuste clasificatorio de algunos elementos para facilitar las tareas de observación de la escucha televisivas de los diferentes países para su posterior análisis comparado.

Así, el indicador Tipo de sonido que hace referencia en la plantilla inicial a la sensación auditiva que produce en nosotros el fenómeno físico originado por las vibraciones de los cuerpos fue desglosado en una serie de elementos constitutivos internos. Esta clasificación inicial fue sometida a una revisión que permitiera una observación más objetiva, y tuvo como resultante los 9 items mostrados en la plantilla final. Del resto de elementos de la plantilla resumimos en este apartado únicamente las características que sufrieron modificaciones, el resto pueden localizarse en la plantilla resultante:

El elemento Ritmo, considerado como ordenación de sonidos en el tiempo distinguía entre ritmos binarios, ternarios, ritmos de subdivisión binaria y ternaria y amalgamas.

Intensidad, considerada como la cualidad que permite distinguir entre sonidos fuertes y débiles en los que se incluía la dinámica como formas de variación de la intensidad.

Tonalidad, considerada como la organización musical que se define por el orden de los intervalos dentro de la escala de los sonidos.

Modo, como correspondiente a los tipos de escala mayor, menor y otras.

Estructura musical, (morfología o arquitectura musical) considerada como sinónimo de forma musical, es decir, la organización de las ideas musicales. 
Simultaneidad sonora, se refiere a las predominancias, planos sonoros, texturas y relaciones entre figura y fondo de la obra considerada.

\section{Juicio de expertos}

El primer proceso de validación se realizó mediante la evaluación de expertos. Estos formaban parte de dos ámbitos de conocimiento necesarios para la realización del estudio: el metodológico y el de contenido. Los expertos del ámbito metodológico trabajaron en la depuración y ajuste de la toma de decisiones de aspectos relacionados con las mediciones y la metodología de la investigación. El segundo grupo, expertos internacionales en el ámbito del contenido de la investigación, la Educación Musical, tenían una función asignada para la depuración y toma de decisiones de los aspectos relacionados con la temática: la música y sus características en el contexto latinoamericano. Para la realización de esta primera validación, todos y cada uno de los expertos de Educación Musical tuvieron que tomar una serie de observaciones sobre la programación a analizar utilizando para ello, la plantilla piloto diseñada. Del resultado de esta primera observación, el primer grupo de expertos se encargó de detectar los desajustes metodológicos (duplicidades, variables incompletas, mal delimitadas, etc.). Una vez ambos grupos habían hecho su labor, se realizó una sesión de validación conjunta de tal manera que pudieran establecer soluciones metodológicas acordes al contenido.

Los problemas y soluciones fueron los siguientes:

\section{Aspectos metodológicos:}

1. Nivel de Análisis: Las escuchas realizadas presentaban longitudes diferentes (canciones completas, cortinillas, etc.) lo que no permitía realizar análisis globales. Obviamente, no puede tratarse al mismo nivel una cortinilla de 2 segundos frente a una canción de 3 minutos. Era necesario realizar esa distinción o bien uniformar las unidades de análisis. De esa manera, se decide que la unidad de análisis mayor sea la frase, y en caso de ser unidades menores se analizarán también haciéndolo explícito en la cabecera de la plantilla.

2. Muestreo: Surge la polémica de cuál es el objetivo del análisis, llegándose a la determinación de que el objetivo principal es analizar qué es lo que escuchan los niños en los programas infantiles. De lo anterior se deduce que esa escucha hace referencia a todo tipo de sonido, por lo que se hace necesario establecer el proceso de muestreo, que afectará al total de la programación considerada para el análisis. Así se decide realizar un corte cada 45 segundos durante toda la emisión del programa y analizar el sonido que se produce en ese momento hasta un máximo de 5 segundos. En caso de que el sonido sea musical, se analizará la frase que esté teniendo lugar durante ese corte. De no existir frases, se analizará la estructura que se trate indicándolo en la cabecera. Se realizan excepciones en lo referente a las canciones, en las que se analizarán la primera y la última frase además de los cortes establecidos cada 45 segundos.

3. Plantilla: Debido a la dificultad de identificar, en las unidades de análisis, algunas de las variables consideradas en la plantilla se decide añadir en todas las variables una categoría que indique que no se ha podido identificar el nivel de dicha variable. De esta manera se evita dejar la variable en blanco y los cálculos posteriores no se vean afectados.

4. Variables: Se han observado numerosos desajustes a la hora de establecer los perfiles sonoros escuchados debido, principalmente, al carácter temporal de la Música y a la presencia de cualidades sonoras interconectadas y dependientes en el todo. Por todo ello los niveles considerados en las diferentes variables no han sido totalmente excluyentes, por lo que debe realizarse un estudio pormenorizado de cada una de las variables y sus niveles de manera que se 
asegure que el investigador que realice la escucha señalé todas las variables y uno y solo uno de los niveles en cada una de ellas.

5. Validación de la escucha: Debido a la dificultad de llevar a cabo las escuchas realizadas para el estudio piloto, se han producido algunos desacuerdos en cuanto a las características escuchadas. Así, se determina que es preciso utilizar escuchas cruzadas que validen los resultados. En caso de desacuerdo podrá establecerse un consenso o buscar a un tercer juez.

Aspectos de contenido:

1. Se sustituye el término música por el de sonido, puesto que lo que se está buscando son las características de la banda sonora de la programación infantil, y esto incluye música y cualquier otra tipología de sonido.

2. La variable piloto Tipo de sonido se divide ahora en dos variables: sonido musical y sonido no musical.

3. La variable $\operatorname{Voz}$ se reduce únicamente al género que produce el sonido cuando este es cantado, incluyendo la posibilidad de grupos vocales, y sonido instrumental.

4. La variable Ritmo se reestructura en Métrica y Rítmica considerando únicamente los niveles binario y ternario, $\mathrm{y}$ dando opción a otras variaciones.

5. El Tipo de Comienzo incluye ahora también la categoría Acéfalo.

6. Aparece una nueva variable, Dinámica, antes incluida en la variable Intensidad.

7. En la variable Agógica se añade la categoría sin variación.

8. La variable Género y Estilo que contaba con 10 niveles más algunos subniveles, pasa a resumirse en la nueva plantilla en 4 niveles, que agrupan los principales géneros y estilos y posibilita la existencia de combinaciones. Se hace también explícito el carácter propio o externo del género tradicional en caso de darse.

9. Las variables Tono y Modo de la plantilla piloto se agrupan en una nueva variable denominada Organización Sonora.
10. La variable Estructura Musical sufre varios y significativos cambios. Por un lado, las referencias a la estructura musical pasan a ser unidades de análisis de la muestra seleccionada, por lo que se indican en la cabecera de la nueva plantilla. El resto de niveles desaparecen, a excepción del nivel denominado Resoluciones que pasa a ser considerado como una nueva variable denominada Cadencias.

11. En cuanto a la variable denominada Simultaneidad Sonora se subdivide, en la nueva plantilla en dos nuevas variables: Textura sonora y Plano Sonoro.

\subsection{Segunda Plantilla ${ }^{[1]}$}

A partir de los resultados detallados en el apartado anterior, se rediseña el instrumento, a partir de las siguientes variables de análisis:

\section{0 . SIN SONIDO.}

1. SONIDO NO MUSICAL- Este indicador hace referencia al sonido no mensurable con parámetros musicales por pertenecer al medio natural o social.

2. SONIDO MUSICAL - Se refiere al conjunto de cualidades sonoras que permiten discriminar y distinguir componentes musicales

3. VOZ- Este indicador hacer referencia al sonido producido por el aparato fonador humano.

\section{MÉTRICA Y RÍTMICA}

a. Métrica: Se refiere a la organización jerárquica de medida o niveles temporales que presentan permanencia en el discurso.

b. Rítmica: Se refiere a la distribución de sonidos en el continuum temporal

\section{TIPO DE COMIENZO}

a. Comienzo anacrúsico Se refiere aquel que comienza antes del inicio de la frase b. Comienzo acéfalo. Referido a aquella frase que comienza con silencio

c. Comienzo tético: Se refiere a que la frase comienza a tiempo 
6. DINÁMICA- Hace referencia a lo relacionado con los grados de intensidad de la música.

7. AGÓGICA- Este indicador se refiere a aquellos aspectos de la interpretación relacionados con la duración y por extensión en el tempo.

8. GÉNERO Y ESTILO.

a. Género musical Este indicador hace referencia a la categoría que reúne composiciones musicales que comparten distintos criterios de afinidad

$b$. Estilo: Se refiere a la forma del discurso y la manera particular en la cual una obra es ejecutada.

9. ORGANIZACIÓN SONORA- Este indicador se refiere a la sintaxis musical, organización tonal (mayor o menor) o modal de la música occidental.

10. CADENCIA - Hace referencia al proceso de caída hacia un centro tonal. Las diferentes cadencias se clasifican en con- clusivas, si generan cierto grado de estabilidad o reposo; y suspensivas, si generan inestabilidad o tensión.

11. MODULACIÓN - Este indicador se refiere en la música tonal, al proceso de pasar de una a otra tonalidad o el resultado de este cambio.

12. TEXTURA SONORA: Este indicador permite comprender la organización espacial y sus articulaciones en el plano de las ejecuciones, de la composición y del análisis. Tipos de textura: i) Monodía; ii) Polifonía: a) Polifonía horizontal; b) polifonía vertical; iii) Melodía acompañada

13. PLANO SONORO Se refiere a los grupos vocales $\mathrm{y} / \mathrm{o}$ instrumentales que intervienen conjuntamente y determinan la situación, ya sea temporal, física o de intención de los distintos sonidos.

El instrumento queda configurado para el estudio piloto de la siguiente manera:

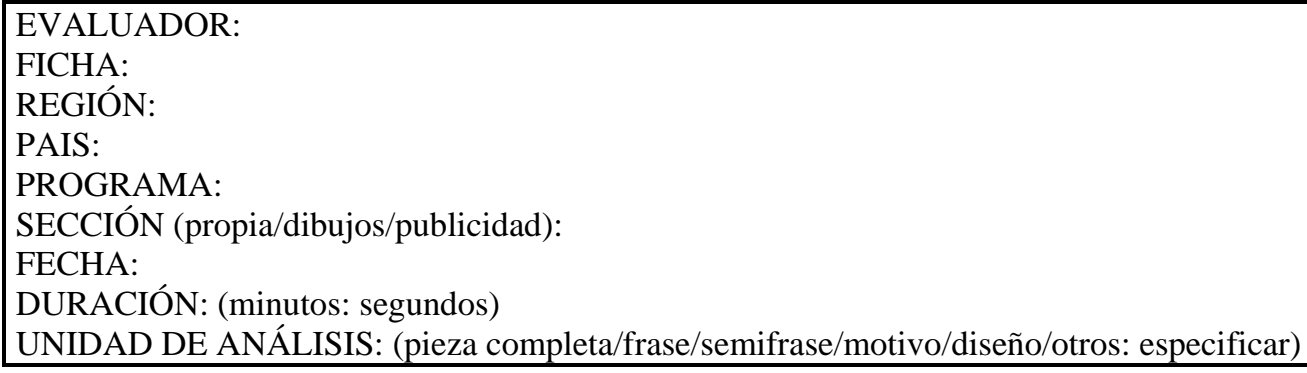

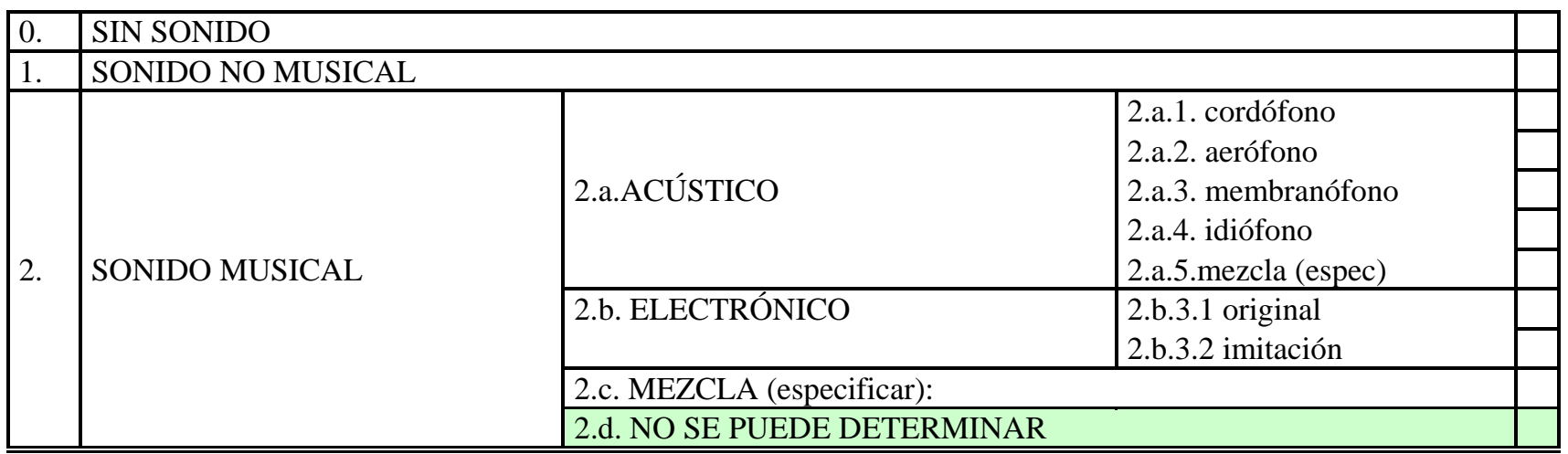


Porta, Amparo y Ferrández, Reina (2009). Elaboración un instrumento para conocer las características de la banda sonora de la programación infantil de televisión. RELIEVE, v. 15, n. 2, p. 1-18.

http://www.uv.es/RELIEVE/v15n2/RELIEVEv15n2_6.htm

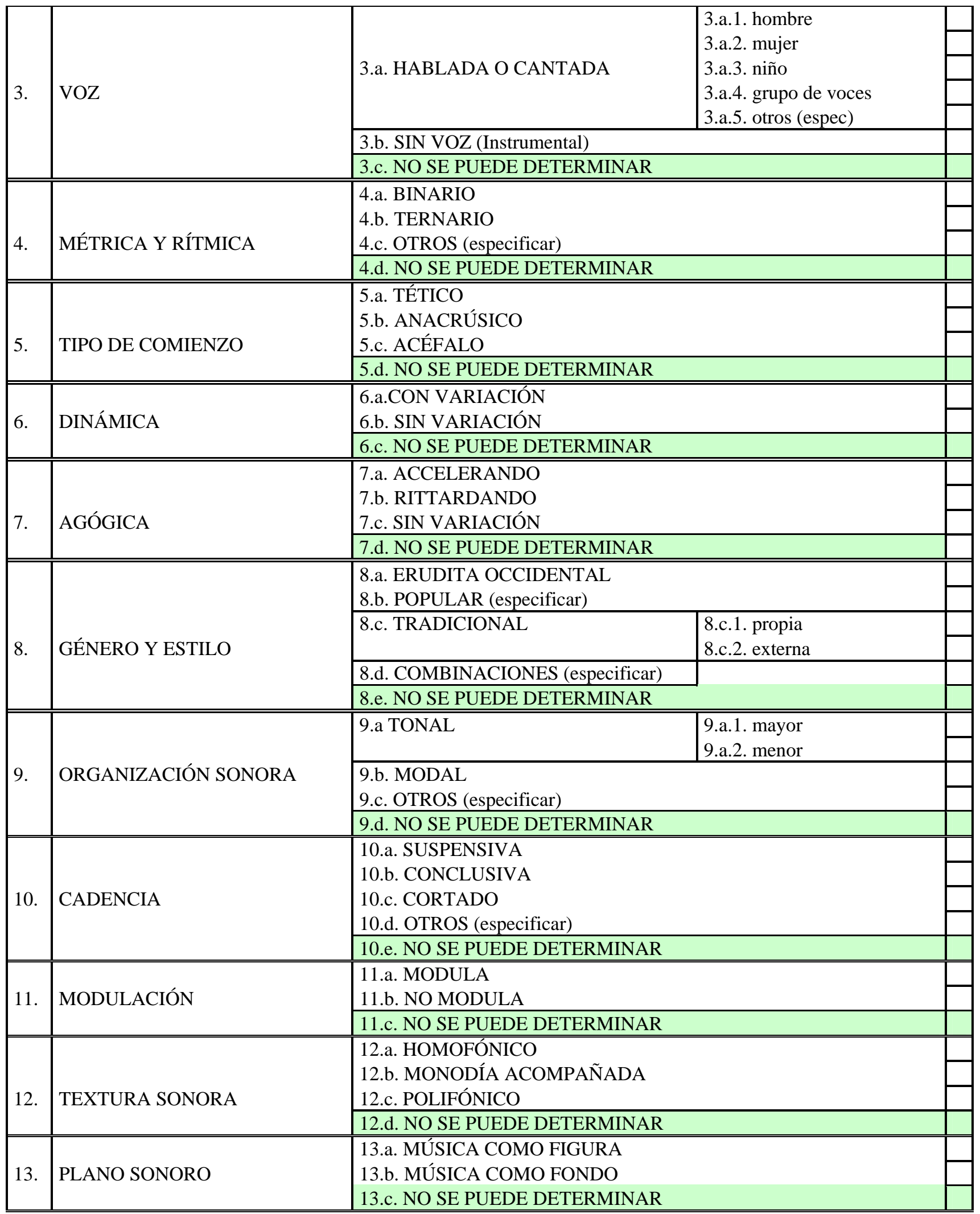




\section{Validación interjueces}

El grupo investigador base está formado por investigadores de Chile, Argentina, Brasil y España y realiza el estudio de las programaciones de televisión de diez cadenas públicas y privadas de los países mencionados. Pero para este segundo paso en la validación, dada la dificultad y laboriosidad de la recogida de la información, se utilizó el juicio cruzado de tres jueces independientes $^{[2]}$ sobre 22 unidades de análisis de un mismo programa. De esta manera, tal y como se realiza en otros estudios (Ramos, 2005) pretendemos avalar la información recogida por dichos jueces a través de la consistencia de sus observaciones y analizamos las discrepancias existentes entre ellos con el fin de mejorar el instrumento inicial.
Para ello se han tomado como programa piloto la serie de española "Los Lunnis" emitida por la segunda cadena de Televisión Española, semana de 15 al 20 de febrero 2008. De este programa hemos extraído algunos elementos musicales representativos de la muestra televisiva de utilidad en las muestras de los países seleccionados: canciones, series de dibujos animados y publicidad. En cuanto a la selección musical interna, hemos tomado como referencia la frase musical por expresar una idea completa, así como sus niveles de significación inferior, es decir, los motivos musicales y los diseños (Zamacois, 1968).

De esta manera, la selección resultante ha sido la siguiente:

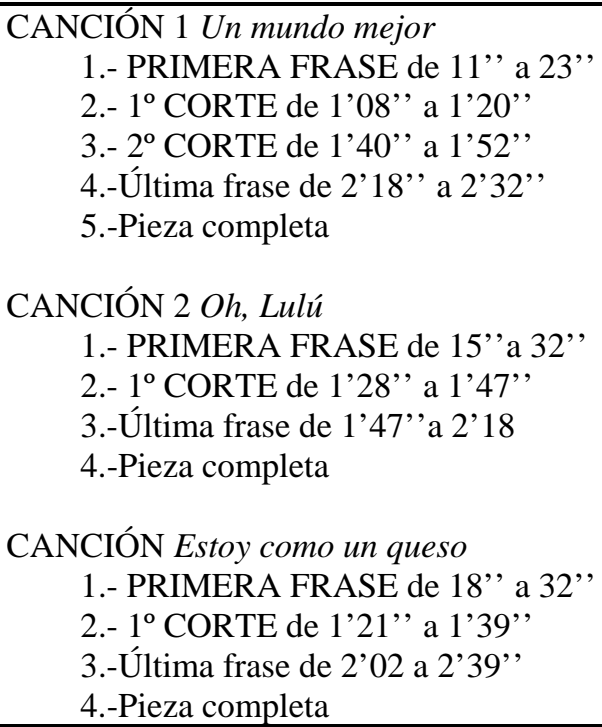

Dadas las características dicotómicas de los datos observacionales recogidos, el índice de concordancia se ha calculado a partir del porcentaje de acuerdos sobre el total de juicios para cada variable. Así, los resultados globales de los juicios para esta fase fueron los siguientes:

Acuerdos totales (Los tres jueces emitieron el mismo juicio): 69'70 \%

Acuerdos parciales (dos de los jueces estuvieron de acuerdo y uno en desacuerdo): $28 ’ 03 \%$

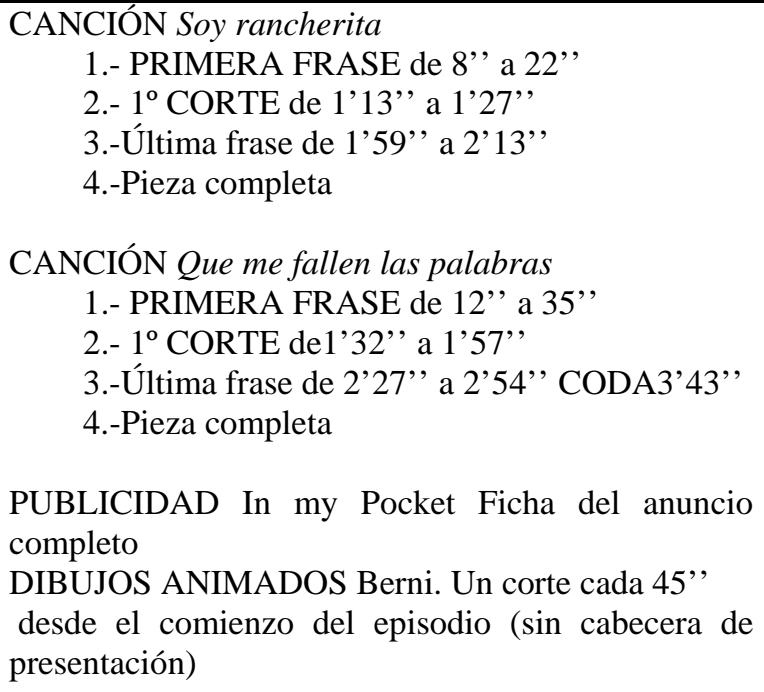

PUBLICIDAD In my Pocket Ficha del anuncio completo

DIBUJOS ANIMADOS Berni. Un corte cada 45, desde el comienzo del episodio (sin cabecera de presentación) 
Además, creemos destacado señalar, que en ningún caso fue señalada la categoría "no se puede determinar" por lo que, en principio, las unidades de análisis pueden considerarse con significado y estructura suficiente.

Sin embargo, a este respecto, debe tenerse en cuenta que el tamaño de las unidades utilizadas no es equiparable: una pieza completa requería de una capacidad de síntesis de cada evaluador que podría resultar diferente en cuanto se añade un componente subjetivo mucho mayor que cuando la unidad era la frase musical.

Es por ello, que decidimos analizar también por separado ambos aspectos con el fin de determinar si la plantilla podría resultar igualmente válida independientemente del tamaño de la unidad muestral.

De esta forma, para las 16 unidades de análisis mínimas consideradas (frases musicales), los resultados han sido:

Acuerdos totales (tres acuerdos): 69’27\%
Acuerdos parciales (dos acuerdos y un desacuerdo): 28'65\%

Desacuerdos (tres desacuerdos): 2’08\%

Y para las unidades máximas (piezas completas):

Acuerdos totales (tres acuerdos): 70’83\%

Acuerdos parciales (dos acuerdos y un desacuerdo): 26'39\%

Desacuerdos (tres desacuerdos): 2'78\%

Aplicando la prueba Chi-cuadrado no se observan diferencias significativas para un nivel de confianza del 99\%, por lo que parece quedar claro que la plantilla funciona de una manera prácticamente idéntica independientemente del tamaño de la unidad de análisis utilizada.

El siguiente aspecto a considerar es el nivel individual de cada variable, es decir, cuál ha sido el comportamiento de cada variable en el juicio que ha emitido cada juez. Dado que no se han encontrado diferencias significativas entre los tamaños de unidades de análisis, análisis se realizará globalmente:

\begin{tabular}{|l|c|c|c|c|l|}
\hline \multicolumn{1}{|c|}{ Variable } & $\begin{array}{c}\text { \% Acuerdo } \\
\text { Total }\end{array}$ & $\begin{array}{c}\text { \% Acuerdo } \\
\text { Parcial }\end{array}$ & $\begin{array}{c}\text { \% Sin } \\
\text { acuerdo }\end{array}$ & $\begin{array}{c}\text { Grado de } \\
\text { concordancia }\end{array}$ & \multicolumn{1}{|c|}{ Variable } \\
\hline Tipo de sonido & 27,27 & 54,55 & 18,18 & 63,64 & Equívoca \\
\hline Voz & 63,64 & 27,27 & 9,09 & 81,82 & Equívoca \\
\hline Métrica y rítmica & 81,82 & 18,18 & 0,00 & 93,94 & Inequívoca \\
\hline Tipo de comienzo & 72,73 & 27,27 & 0,00 & 90,91 & Difusa \\
\hline Dinámica & 100,00 & 0,00 & 0,00 & 100,00 & Inequívoca \\
\hline Agógica & 63,64 & 36,36 & 0,00 & 87,88 & Difusa \\
\hline Género y estilo & 40,91 & 59,09 & 0,00 & 80,30 & Difusa \\
\hline Organización sonora & 81,82 & 18,18 & 0,00 & 93,94 & Inequívoca \\
\hline Cadencia & 31,82 & 68,18 & 0,00 & 77,27 & Difusa \\
\hline Modulación & 90,91 & 9,09 & 0,00 & 96,97 & Inequívoca \\
\hline Textura sonora & 81,82 & 18,18 & 0,00 & 93,94 & Inequívoca \\
\hline Plano sonoro & 100,00 & 0,00 & 0,00 & 100,00 & Inequívoca \\
\hline
\end{tabular}

De este modo, en cuanto al porcentaje de acuerdos (tres primeras columnas), se pueden apreciar tres grupos de variables que han funcionado de manera diferente:
En primer lugar, encontramos el grupo de variables que denominaremos inequívocas, es decir, aquellas en el que el grado de acuerdo ha sido total en un número de casos superior al $80 \%$. Aquí encontramos un total de 6 variables (50\%) que son:”Métrica y 
Rítmica”, "Dinámica”, “Organización Sonora”, "Modulación”, “Textura Sonora” y "Plano Sonoro". Estas variables, en principio, funcionan muy adecuadamente, por lo que no es necesaria su revisión.

En segundo lugar, tenemos un grupo de variables que denominaremos equívocas. Se trata del "Tipo se Sonido" y la "Voz". Son los únicos casos en los que se han detectado algunos desacuerdos totales, sobre todo en el caso de la variable "Tipo de sonido". Aquí, el hecho de que, además, el porcentaje de acuerdos parciales sea muy superior al de acuerdos totales, hace imprescindible su revisión. El caso de la variable "Voz" también se hace necesario un análisis más pormenorizado en tanto en cuanto presenta valores de acuerdo extremos.

Por último, encontramos el grupo de variables en las que, si bien ya no hay más desacuerdos totales, los porcentajes entre los acuerdos totales y parciales son equilibrados o incluso, en algunos casos, superior en los parciales. A este grupo lo denominaremos difusas. Se trata de cuatro variables (33’3\%) “Tipo de Comienzo”, “Agógica”, “Género y Estilo” y “Cadencia”. En estos casos sería recomendable una revisión con el fin de mejorar los grados de acuerdo en las categorizaciones.

En lo que se refiere al grado de concordancia (última columna), puede observarse que siempre es muy elevado y superior al $80 \%$, lo que de nuevo avala la fiabilidad de la plantilla. Sin embargo, destacan las variables “Tipo de Sonido" y “Cadencia” por cuanto la concordancia es significativamente menor que en el resto de variables. Esto redundaría en la necesidad de una revisión en ambos casos, si bien, ya era patente dicha necesidad en el caso del "tipo de Sonido".

Tipo de sonido: La ambigüedad del ítem requiere una mejor definición, por ello proponemos distinguir entre sonido acústico, electrónico, híbrido y añadir un nuevo item, el de timbre, para liberar el indicador de tím- brica de su condición de cualidad acústica excluyente. De este modo conseguiremos una mejora sustancial en nuestro instrumento de medida, que tiene en este apartado uno de los indicadores relevantes de las cualidades sonoras del entorno televisivo. Sin embargo, queremos precisar que es muy difícil por la escucha reconocer la naturaleza de determinados sonidos, excepto en los casos extremos de los sonidos electrónicos de imitación de muy baja calidad y los electrónicos originales. En cualquier caso, la detección de estos extremos ya es un elemento valioso a determinar en nuestro análisis del entorno sonoro. Conocer la naturaleza, acústica o electrónica de imitación u original del sonido musical televisivo es un factor determinante, especialmente, cuando existe la sospecha de que el sonido imitado se ofrece de forma fraudulenta como simulacro, sin referencia a original.

Las cadencias: Se propone como solución sustituir el ítem por "Terminación en cadencia” con tres opciones Si, No, Cortado. En caso afirmativo colocar las tres últimas notas o acordes de la selección analizada. Tipo de comienzo: especificar las figuras rítmicas de comienzo en el primer compás, sólo en el caso en que sea necesaria la revisión.

Agógica: computar cualquier variación de la velocidad, independientemente de su grado o duración en la obra, por ello recomendamos, si es necesario, utilizar el apoyo de un metrónomo en el análisis

Genero estilo: este indicador tiene un componente poco objetivable en la medida en que depende de los referentes y esquemas clasificadores del observador. Los resultados de la investigación servirán para recoger las grandes selecciones estilísticas y, de forma especial, nos acercará a la elaboración de otro instrumento evaluador pendiente: la selección estilística de la banda sonora de la programación infantil de televisión y su relación con el patrimonio sonoro propio y de la cultura hispana. 


\section{A modo de conclusión}

Estas líneas suponen un intento de sistematización del conocimiento del entorno más cotidiano de la infancia actual, la televisión. A pesar de la importancia y repercusiones como elemento configurador cultural, comunicativo y patrimonial de la cultura contemporánea no existen instrumentos de observación para conocer qué escuchan los niños en la televisión y cuáles son los elementos musicales que la televisión maneja. De igual modo buscamos la respuesta a cuáles de ellos tienen como referente los contenidos musicales de la proximidad de la cultura propia o ajena y, como consecuencia, cuales son los efectos de la globalización en materia musical y sonora.

El proyecto de investigación sobre la escucha del entorno sonoro cotidiano de la cultura hispana tiene carácter amplio, se compone de diferentes estudios comparados sobre las bandas sonoras de las programaciones televisivas de los diferentes países participantes. En este artículo hemos presentado el proceso seguido en la creación de una herramienta, sujeta todavía a múltiples revisiones, correcciones y matizaciones, de carácter cuantitativo y empírico: la elaboración un instrumento de observación para conocer las características de la escucha de la banda sonora de la programación infantil.

Todo ello, desde una mirada educativa que observa y cuestiona el entorno sonoro con intención de tener argumentos para modificar las políticas culturales en materia televisiva, resituar y dar valor lingüístico, comunicativo y patrimonial a la Música en la educación y proponer modificaciones en los diseños curriculares presentes y futuros.

\section{Bibliografía}

Adorno, T.W. (1971). Sociologie de la musique. Musique en $\mathrm{Jeu}, \mathrm{N}^{\circ} 2$, Paris, Seuil, Mars, pp. 5-13.
Aguaded, J.I. (2005). Enseñar a ver la televisión: una apuesta necesaria y posible. Comunicar 25, Huelva, pp. 51-55.

Atienza, R. (2004). L'identité sonore: une variable essentielle dans la configuration urbaine. Grenoble: CRESSON, pp. 161166.

Augoyard, J.F.; Leroux, M. (1989). Journee d'etude nationale: Les Faiseurs de bruit (casset). Grenoble, CRESSON / EUTERPES.

Augoyard, J.F., Torgue, H. (Eds.) (1995). A l'écoute de l'environnement. Répertoire des effets sonores. Marsella: Parenthèses, 174 p.; pp. 6-7.

Benjamin, W. (1973). La obra de arte en la época de su reproductibilidad técnica, en Discursos interrumpidos I, Prólogo, traducción y notas de Jesús Aguirre. Madrid: Taurus.

Boletín Oficial del Estado (2003). Real Decreto 943/2003, de 18 de julio, BOE del 31. España

Boletín Oficial del Estado (2006a). Ley Orgánica 2/2006) Título II, cap 1, artículo 71,76 y 77. España

Boletín Oficial del Estado (2006b). Real Decreto 1513/2006, de 7 de diciembre. España.

Burnard, P. (2008/2006). Reflective Practices in Arts Education. Dordrecht: Springer.

Cage, J. (1961). Silence. Middletown: Wesleyan University Press.

De Moragas, M. (1991). Teorías de la comunicación (5 ${ }^{\mathrm{a}}$ ed.). Barcelona: Gustavo Gilli.

Del Río, M y Román, M. (2005). Programación infantil de televisión: Orientaciones y contenidos prioritarios. Madrid: Ministerio de trabajo y asuntos sociales, Instituto Oficial de Radio y Televisión.

Del Río, P.; Álvarez, A. y Del Río, M. (2004). Pigmalión. Informe sobre el impacto de la televisión en la infancia. Madrid: Fundación Infancia Aprendizaje.

Eco, U. y Cantarell, F. S. (1978). La estructura ausente: Introducción a la semiótica. Madrid: Lumen.

Ferrés, J. (1994, 1995). Televisión y educación. Barcelona: Paidós. 
García Muñoz, N. (1997). Los hábitos televisivos del niño frente al televisor en el hogar en Revista de Estudios en Comunicación 3, Bilbao.

Gómez-Ariza, C. (2000). Determinants of musical representation. Cognitiva, vol. 12, no. 1, pp. 89-110.

Hauser , A. (1963). Historia social de la literatura y el arte. (Tr. por A. Tovar y F. P. Varas-Reyes). Madrid : Guadarrama.

Koivusilta, L. K., Rimpelä, A. H. and Rimpelä, M. K. (1999). Health-related lifestyle in adolescence - origin of social class differences in health?. Health education research, vol. 14, no. 3, pp. 339-355.

Morley, D. (1996). Televisión, audiencias y estudios culturales. Buenos Aires: Amorrotu.

Orozco, G. (1996). Miradas latinoamericanas a la televisión. México: Universidad Iberoamericana.

Ostbye, T. (1993). Food and Nutrition in Canadian Prime-Time Television Commercials. Canadian Journal of Public HealthRevue Canadienne De Sante Publique, vol. 84, no. 6, pp. 370-374.

Peterson, J. L. y Newman R. (2000). Helping to curb youth violence: The APA-MTV Warning Signs initiative. Professional Psychology-Research and Practice, vol. 31, no. 5, pp. 509-514.

Pintado, J. (2006). Los medios de comunicación y la construcción de la identidad adolescente. ZER, vol. 11, no. 21, pp. 11-22.

Porta, A. (2004a). Contenidos musicales buscan curriculum. Comunicación y pedagogía: Nuevas tecnologías y recursos didácticos, pp. 31-36.

Porta, A. (2004b). Musical expression as an exercise in freedom. ISME 26 International Society for Music Education World Conference. Tenerife, España.

Porta, A. (2007). Hablemos de educación, hablemos de música. Eufonía: Didáctica de la música, pp. 87-98.

Ramos, G. (2005). Elementos para el diseño de planes de evaluación de programas de teleformación en la empresa. RELIEVE, $v$. 11 ,

n.
www.uv.es/RELIEVE/v11n2/RELIEVEv11 n2 5.htm.Consultado el 2/2/09.

Register, D. (2004). The effects of live music groups versus an educational children's television program on the emergent literacy of young children, Journal of music therapy, vol. 41, no. 1, pp. 2-27.

Reig, R. (2005). Televisión de calidad y autorregulación de los mensajes para niños y jóvenes. Comunicar 25, Huelva, pp. 63-70.

Reyes, J (2006). Perpendicularidad entre arte sonoro y música. Universidad de $\mathrm{Pa}$ lermo. Facultad de diseño y comunicación.

Schaeffer, P. (1966). Traité des objets musicaux. París: Seuil.

Schafer, M. (1977). The tuning of the world. Toronto: McClelland and Steward.

Silverstone, R. (1996). Televisión y vida cotidiana. Buenos Aires: Amorrortu.

Vallejo Nágera, A. (1987). Mi hijo ya no juega, solo ve la televisión. Madrid: Temas de hoy.

Vargas, E. y Barrena, F. (2003). Influencia de los programas televisivos en el contenido sexual sobre el comportamiento de los adolescentes. Bogotá: Comisión Nacional de Televisión CNTV.

Vygotski, L. (1981). The instrumental method in psychology. En J. Wertsch, The Concept of Activity in Soviet Psychology, pp.134-143. New York: Sharpe.

Ward-Steinman, P. M. (2006). The development of an after-school music program for at-risk children: student musical preferences and pre-service teacher reflections. International Journal of Music Education, vol. 24, no. 1, pp. 85-96.

Zamacois, J. (1986). Curso de formas musicales $\left(10^{\mathrm{a}}\right.$ ed.). Barcelona: Labor

\section{Notas}

[1] Estos indicadores y sus definiciones fueron debatidos y consensuados en el II Encuentro investigador sobre La banda sonora de la televisión infantil y juvenil en el ámbito latinoamericano. Variables, impacto e influencia en el patrimonio sonoro", celebrado en la 
Universidad de LANUS, Buenos Aires (Argentina) entre el 14 y 18 de Mayo de 2008.

[2] Nuestro agradecimiento especial a Jesús Debón, compositor, arreglista y creador de bandas sonoras para diferentes producciones audiovisuales, y a Remigi Morant, profesor del Departamento de Didáctica de la Expresión Musical de la Universidad de Valencia, por su colaboración desinteresada en esta fase.

\section{$\underline{\text { ABOUT THE AUTHORS / SOBRE LOS AUTORES }}$}

Amparo Porta (porta@edu.uji.es): Licenciada en CC de la Educación y Doctora en Teoría de los Lenguajes por la Universidad de Valencia. Profesora Titular de Universidad del Departamento de Educación de la Universitat Jaume I, en el Área de Didáctica de la Expresión Musical. Sus investigaciones se dirigen hacia la comprensión del hábitat sonoro y el imaginario como construcción cultural del niño y el adolescente, la Semiótica de la música aplicada a la educación y la Didáctica de la expresión musical en un contexto cambiante. Su dirección postal es: Departamento de Educación, Campus de Riu Sec. Universidad Jaume I. 12071-Castellón (España). Buscar otros artículos de esta autora en Scholar Google

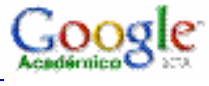

M ${ }^{a}$ Reina Ferrández Berrueco (ferrande@edu.uji.es).Licenciada y doctora en Filosofía y Ciencias de la Educación (sección CC. De la Educación) por la Universidad de Valencia. Profesora Titular del Departamento de Educación de la Universitat Jaume I en el Área de Métodos de Investigación y Diagnóstico en Educación. Centra su línea de investigación en evaluación de la calidad así como en aspectos metodológicos asociados a la investigación educativa. Es la autora de contacto para este artículo. Su dirección postal es: Departamento de Educación, Campus de Riu Sec. Universidad Jaume I. 12071-Castellón (España). Buscar otros artículos de esta autora en Scholar Google Google 
Porta, Amparo y Ferrández, Reina (2009). Elaboración un instrumento para conocer las características de la banda sonora de la programación infantil de televisión. RELIEVE, v. 15, n. 2, p. 1-18.

http://www.uv.es/RELIEVE/v15n2/RELIEVEv15n2_6.htm

\section{ARTICLE RECORD / FICHA DEL ARTÍCULO}

\begin{tabular}{|c|c|}
\hline $\begin{array}{l}\text { Reference / } \\
\text { Referencia }\end{array}$ & 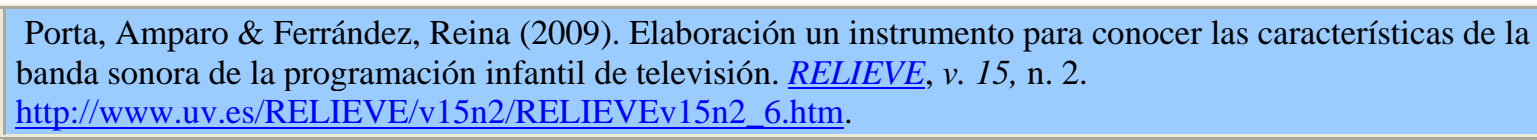 \\
\hline Title / Título & $\begin{array}{l}\text { Elaboración un instrumento para conocer las características de la banda sonora de la programación infantil } \\
\text { de televisión. [Development of an instrument for analyzing the characteristics of the soundtracks of chil- } \\
\text { dren's television programmes]. }\end{array}$ \\
\hline Authors / Autores & Porta, Amparo \& Ferrández, Reina \\
\hline Review / Revista & RELIEVE (Revista ELectrónica de Investigación y EValuación Educativa), v. 15, n. 2 \\
\hline ISSN & $1134-4032$ \\
\hline $\begin{array}{l}\text { Publication date / } \\
\text { Fecha de publica- } \\
\text { ción }\end{array}$ & $\begin{array}{l}2009 \text { (Reception Date: } 2009 \text { May 3; Approval Date: } 2009 \text { October 20; Publication Date: } 2009 \text { October } \\
\text { 24). }\end{array}$ \\
\hline Abstract / Resumen & $\begin{array}{l}\text { Television is one of the most important elements in the construction of the musical and audible reality of } \\
\text { children and teenagers. It offers a selected and edited version of a musical environment, with an indisput- } \\
\text { able level of communicative and patrimonial influence. This paper is part of a research project that sets out } \\
\text { to know what children are listening to in children's television programming, in a sample of Latin American } \\
\text { television coverage. The general objective has been to establish the parameters of everyday listening experi- } \\
\text { ences and to create an instrument which is able to determine the objective elements of listening environ- } \\
\text { ments through their most notable medium: the soundtrack of children's television programmes. Here we } \\
\text { present the creation and validation process of such an instrument. An important element is the consensus } \\
\text { process, since we attempt to validate the instrument within the Latin-American context; decision-making } \\
\text { because of the inherent characteristics of the music as variable analysed, since sound develops over time, } \\
\text { and this makes an accurate classification of it very difficult. } \\
\text { La televisión es un elemento destacado en la construcción de la realidad musical y sonora del niño y del } \\
\text { adolescente que ofrece, como totalidad, una versión seleccionada y editada del entorno musical. El artículo } \\
\text { que presentamos forma parte de un proyecto de investigación que trata de conocer qué escuchan los niños en } \\
\text { la programación infantil de televisión en una muestra latinoamericana. El objetivo general ha sido establecer } \\
\text { los parámetros de la escucha televisiva y determinar sus elementos objetivos. Lo que presentamos en este } \\
\text { artículo es el proceso de elaboración y validación de dicho instrumento. El proceso ha tenido como elemen- } \\
\text { tos generadores el consenso por el ámbito de aplicación con validez latinoamericana. la toma de decisiones } \\
\text { por la naturaleza de la música como variable de análisis, y las características del sonido, cuyo desarrollo en } \\
\text { el tiempo requiere formas específicas de clasificación }\end{array}$ \\
\hline $\begin{array}{l}\text { Keywords / Des- } \\
\text { criptores }\end{array}$ & $\begin{array}{l}\text { Soundtrack, quantitative analysis, validation, observation, classification, television, listening } \\
\text { Banda sonora, análisis cuantitativo, validación, observación, clasificación, televisión, escucha. }\end{array}$ \\
\hline $\begin{array}{l}\text { Institution / Insti- } \\
\text { tución }\end{array}$ & Universidad Jaume I (Castellón, España). \\
\hline $\begin{array}{l}\text { Publication site / } \\
\text { Dirección }\end{array}$ & http://www.uv.es/RELIEVE \\
\hline Language / Idioma & Español (Title, abstract and keywords in English) \\
\hline
\end{tabular}

\section{RELIEVE}

Revista ELectrónica de Investigación y EValuación Educativa E-Journal of Educational Research, Assessment and Evaluation

$$
\text { [ ISSN: 1134-4032 ] }
$$

(C) Copyright, RELIEVE. Reproduction and distribution of this articles it is authorized if the content is no modified and their origin is indicated (RELIEVE Journal, volume, number and electronic address of the document).

(C) Copyright, RELIEVE. Se autoriza la reproducción y distribución de este artículo siempre que no se modifique el contenido y se indique su origen (RELIEVE, volumen, número y dirección electrónica del documento). 